\title{
Malignant Hemangiopericytoma of the Bladder: Case Report and Literature Review
}

\author{
Anvi Raina ${ }^{1 *}$, Louis Spencer Krane ${ }^{2}$, Michael Benjamin Gentry ${ }^{3}$, Majid Mirzazadeh ${ }^{2}$ \\ ${ }^{1}$ Wake Forest University School of Medicine, Winston-Salem, NC, USA \\ ${ }^{2}$ Department of Urology, Wake Forest University Baptist Medical Center, Winston-Salem, NC, USA \\ ${ }^{3}$ Department of Pathology, Wake Forest University Baptist Medical Center, Winston-Salem, NC, USA
}

\begin{abstract}
Received: August 03, 2015; Accepted: August 25, 2015; Published: September 20, 2015
*Corresponding author: Anvi Raina, Wake Forest University School of Medicine, 1 Medical Center Boulevard, Winston-Salem, NC 27157, USA, Tel: +252-452-9733; Fax: 336-716-5711; E-mail: araina@wakehealth.edu
\end{abstract}

\begin{abstract}
Hemangiopericytoma/Solitary Fibrous Tumor (HPC/SFT) of the bladder is a very uncommon neoplasm of uncertain malignant behavior. 10 previous cases of HPC/SFT in the bladder have been reported in the literature. Only two cases have been malignant. In this manuscript, we present the third malignant HPC/SFT of the urinary bladder and also review the literature for this very rare tumor.

Keywords: Bladder Cancer; Cystectomy; Literature review; Malignant Hemangiopericytoma; Solitary Fibrous Tumor
\end{abstract}

\section{Introduction}

Bladder cancer is the second most common malignancy of the urogenital system with over 74,000 new cases diagnosed each year [1]. Urothelial/transitional cell carcinoma is the predominant histologic subtype, accounting for $90 \%$ of all bladder cancers in the US and Europe [1]. Conversely, we identified only 10 previous cases of bladder HPC/SFTs. Malignant cases are even less common with only two documented cases. In 2002, the world's soft tissue tumor experts reclassified HPC under soft tissue tumors attributing to its fibroblastic rather than pericytic origin [2]. First described in 1942 by Stout and Murray, HPC has been found throughout the body and makes up of $2.5 \%$ of all soft tissue tumors $[3,4]$. In the genitourinary system, the kidney has the highest prevalence of HPC/SFTs (approximately 50 reports) with fewer cases noted in the prostate and ureter $[5,6,7]$. Although HPC has been associated with a history of trauma, prolonged steroid use, and hypertension, a definitive correlation does not exist [8]. The rarity of this bladder mass makes it's behavior unpredictable and treatment and clinical follow up remain uncertain. This report presents a case of bladder HPC/SFT and provides a literature review.

\section{Case Report}

A 56 year-old African American male with a history of hypertension, chronic back pain, and alcoholism presented with 2 episodes of painless gross hematuria followed by urinary retention. A urine culture showed no growth. No cytology specimen was obtained. A contrasted Computed Tomography (CT) scan was obtained and demonstrated an enlarged prostate with a prominent median lobe and evidence of an intravesical bladder neck mass without lymphadenopathy (Figure 1).

Follow up cystoscopy clearly showed tan, friable bladder neck mass. After transurethral resection, pathology identified a solitary fibrous tumor with diffuse mitotic features concerning for malignancy. Due to these features and the site of tumor being the trigone and bladder neck, a partial cystectomy was unfeasible. The patient elected to undergo a radical cystoprostatectomy with ileal conduit diversion. A 6.5 X 4 X $4.5 \mathrm{~cm}$ butterfly shaped tanwhite solid mass at the junction of the bladder neck was present without invasion into the prostate (Figure 2).

Histologically, this was a spindle cell neoplasm composed of hypercellular sheets of atypical cells with transition areas of less cellularity. The hypercellular areas had cells with enlarged, hyperchromatic, nuclei with minimal cytoplasm and increased mitoses (up to 13 per 10 high power fields) (Figure 3a). The less cellular areas were characterized by scattered, thin-walled,

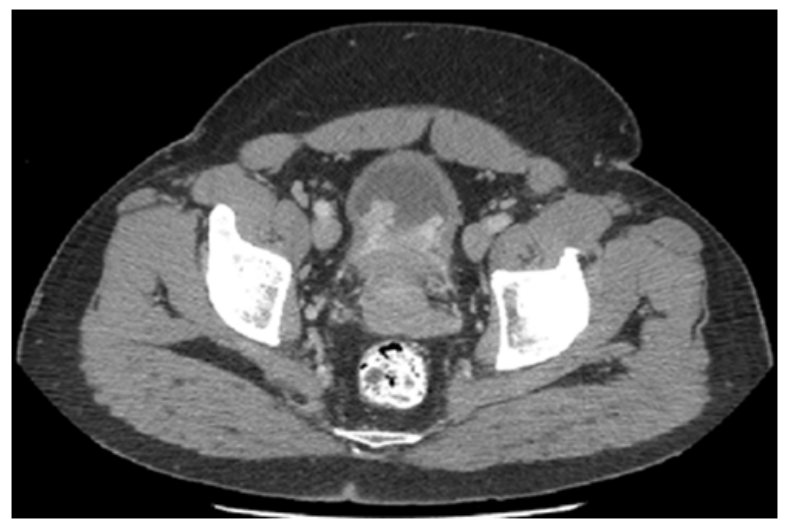

Figure 1: CT with contrast demonstrating bladder neck mass and enlarged prostate 
ectatic vessels with irregular branching and spindled-to-oval cells with bland nuclei, scarce mitoses and variable amounts of sclerotic collagen (Figure 3b). By immunohistochemistry, the neoplastic cells were positive for STAT6 a marker highly sensitive and specific for the NAB2-STAT6 gene fusion found in SFTs. Additionally, the tumor was positive for BCL2 and CD34 and negative for S100, cytokeratin AE1/AE3, desmin, and actin. Due to the elevated mitotic rate ( $>4$ mitoses per $10 \mathrm{hpf}$ ), hypercellularity, and cell atypia, a diagnosis of a malignant HPC/ SFT was rendered. The margins were free of tumor. No adjuvant chemotherapy was administered.

\section{Discussion}

Hemangiopericytoma/Solitary Fibrous Tumor is an exceedingly rare neoplasm of mesenchymal origin that accounts for $2.5 \%$ of all soft tissue tumors [3,4]. Previous literature shows that hemangiopericytomas share various histologic and immunohistochemical features with solitary fibrous tumors [9]. This commonality along with a shared fibroblastic origin prompted a reclassification to HPC/SFT in the World Health Organization meeting in 2002 [2]. These masses have a pseudocapsule and a staghorn vascular network surrounded by spindle cells [10]. The final diagnosis necessitates the presence of CD34, a monoclonal

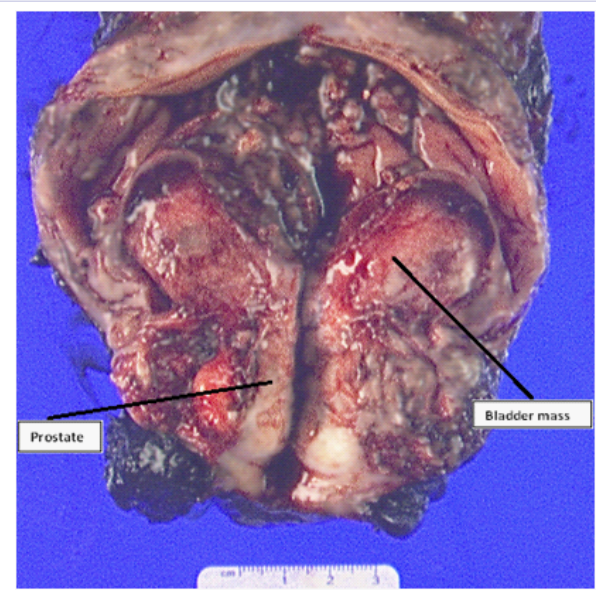

Figure 2: Posterior view of bladder mass (open)
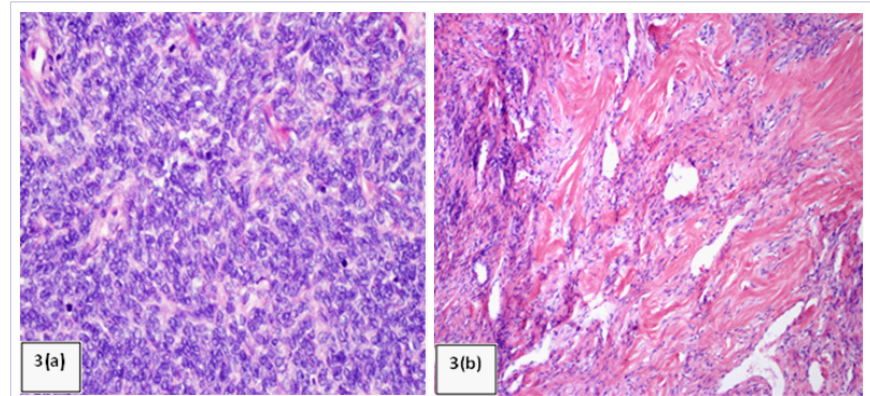

Figure 3: A) Malignant solitary fibrous tumor with densely cellular sheets of atypical, hyperchromatic cells and increased mitotic figures, 400x magnification (a). Areas having typical features of solitary fibrous tumor including thin-walled, branched ectatic vessels and bland-appearing spindled-to-oval cells, 100x magnification (b). antibody against the human hematopoietic progenitor cell antigen [11]. Clinically, HPC/SFTs present as a slow growing mass in middle aged adults of both sexes. The retroperitoneum, pleura, deep soft tissues of proximal extremities, abdominal cavity, trunk, and head and neck are the most common sites[9].The abdominal cavity and retroperitoneum are the two most common sites for malignant HPC/SFTs[3,11] with the lungs, liver, and bones being the primary metastatic sites [12]. Bladder HPC/SFTs are rare; therefore we investigated the available literature for non-bladder HPC/SFTs to identify any trends in management or recurrence. Due to the their solitary, indolent, and locally infiltrative nature, radical resection appears to be the treatment of choice in the CNS, head and neck, abdomen and other sites with chemotherapy and radiation having limited roles $[12,13,14]$.

Two malignant bladder HPC/SFT cases have been reported out of 10 total cases in the literature. The median age of onset for bladder HPC is 50 years old with age ranging from 29 to 72 at the time of diagnosis [15]. HPC in other parts of the body seems to have an equal predisposition towards men and women which is similar in our literature review finding ( 6 female and 4 male). Three patients were diagnosed following episodes of gross hematuria, although five patients described lower urinary tract symptoms including retention, dysuria, and frequency (Table 1). Others presented with symptoms unrelated to the genitourinary tract.

The workup for HPC does not differ from the standard hematuria workup including a cystoscopy and axial imaging of the abdomen and pelvis. The treatment and followup for bladder HPC are uncertain owing to the rarity of the disease. Surgical resection has been as the mainstay of treatment. Other management options included radiation, transurethral resection, partial and radical cystectomy [9]. One transurethral resection and nine cystectomies have been performed previously (Table 1). Transurethral resections may not provide definitive therapy or excise all of the tumor likely due to the non-epithelial origin of the tumor; the sole case also demonstrates recurrence at 2 years $[15,16]$. We believe en bloc resection may provide patients with more durable oncologic outcomes. Adjuvant therapy should be considered in HPCs demonstrating malignant features, although the best modality is yet to be determined [17]. Chemotherapy has been tried in one case with metastasis, but no benefit was demonstrated [17]. Adjuvant radiotherapy appeared to be successful in the case of a 45 year old status post partial cystectomy [18]. We also investigated other genitourinary HPC/ SFTs to guide us regarding adjuvant therapy. In Renal HPC/ SFTs, neither chemotherapy nor radiotherapy has been effective adjuvant modalities $[19,20]$. Given the limited data available in adjuvant therapy, we recommend considering it in a case by case manner in malignant HPC/SFTs.

Long-term follow-up is not available from all previous studies making prognosis uncertain. Outcomes in these studies also appear varied. The longest documented survival without evidence of recurrence is 12 years [21] in a 54 year old man who underwent partial cystectomy for benign HPC/SFT. However, lung metastasis has been reported as far as 9 years 
Table 1: Characteristics and treatments of patients with HPC/SFT, $n=11$ including current case. 1 case treatment unknown.

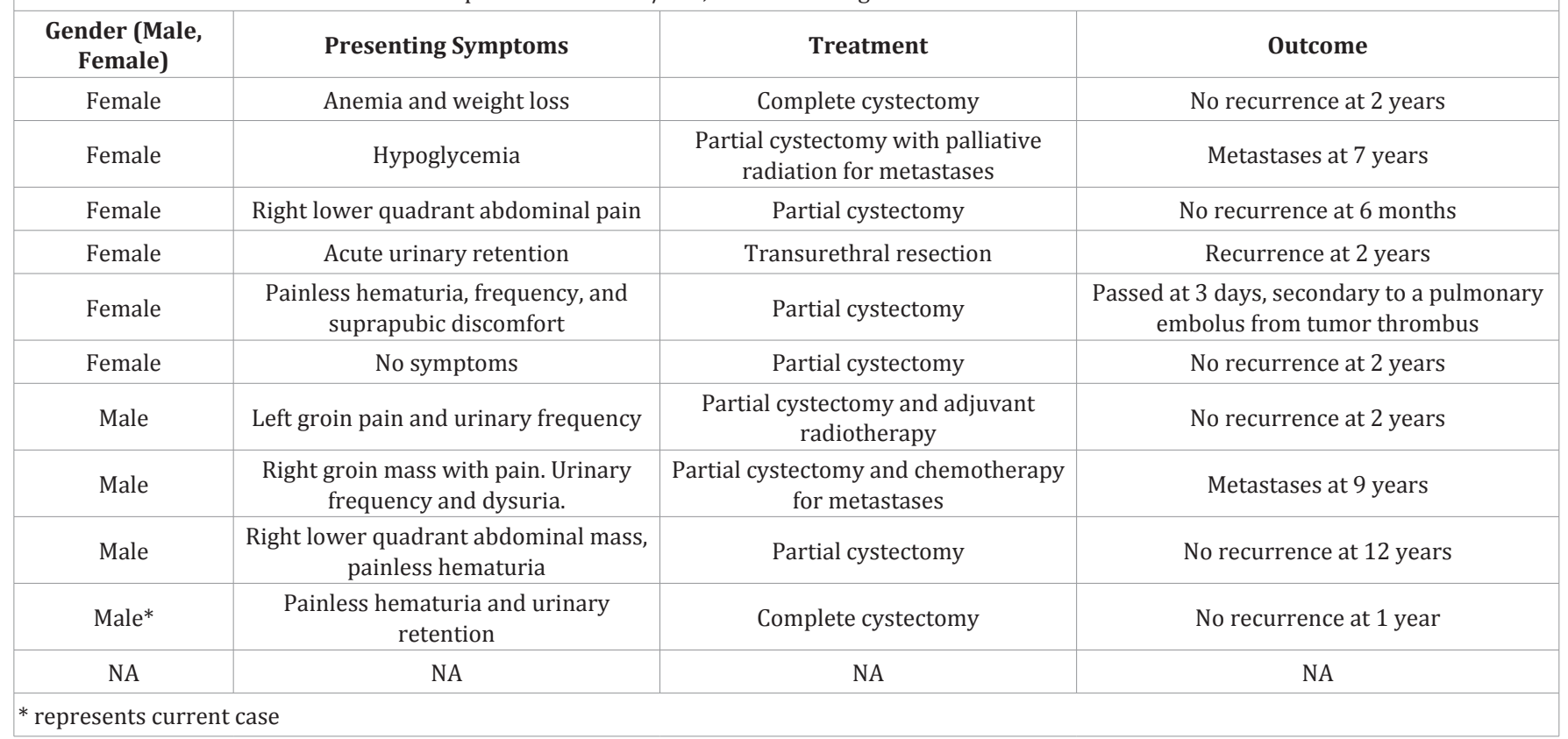

following initial surgery [22]. Therefore, lifelong surveillance is recommended in patients with HPC/SFT of the urinary bladder. Limited experience in bladder HPC/SFTs makes treatment and management uncertain. We present a review of published literature with management recommendation based on previous treatment pathways. Due to the reports of local recurrence with transurethral resections and non-epithelial origin of the tumor, we believe complete resection with partial or complete cystectomy is the choice for best curative rate. For adjuvant therapy, the effectiveness of chemotherapy and radiotherapy remain debatable secondary to the small number of cases.

\section{Conflict of Interest}

There are no conflicts of interests to declare on behalf of any of the authors. Informed Consent was obtained from the patient mentioned in the case report.

\section{References}

1. Siegel RL, Miller KD, Jemal A. Cancer statistics, 2015. CA Cancer J Clin 2015; 65(1):5-29. doi: 10.3322/caac.21254

2. Fletcher CD. The evolving classification of soft tissue tumours: an update based on the new WHO classification. Histopathology. 2006; 48(1): 3-12.

3. Stout AP, Murray MR. Hemangiopericytoma: a vascular tumor featuring Zimmermann's pericytes. Ann Surg. 1942; 116(1): 26-33.

4. Mozafarpour S, Khorramirouz R, Tajali A, Salavati A, Kajbafzadeh AM. Surgically treated bladder hemangiopericytoma/solitary fibrous tumor: report of a 12-year asymptomatic follow-up. Int Urol Nephrol. 2014; 46(3): 483-6.

5. Hu Q, Fang Z, Zhou Z, Zheng J. Renal hemangiopericytoma secondary to refractory hypertension in a child: A case report. Oncol Lett. 2014; 8(6): 2493-2495.

6. Shabaik A. Nonepithelial tumors and tumor-like lesions of the prostate gland. Crit Rev Clin Lab Sci. 2003; 40(4): 429-72.

7. Léger P, Legland PF. [Hemangiopericytoma of the ureter]. Ann Urol (Paris). 1987; 21(4): 285-7.

8. Bokshan SL, Doyle M, Becker N, Nalbantoglu I, Chapman WC. Hepatic hemangiopericytoma/solitary fibrous tumor: a review of our current understanding and case study. J Gastrointest Surg. 2012; 16(11): 2170-6. doi: 10.1007/s11605-012-1947-x.

9. Gengler C, Guillou L. Solitary fibroustumour and haemangiopericytoma: evolution of a concept. Histopathology. 2006; 48(1): 63-74.

10. Argyropoulos A, Liakatas I, Lykourinas M. Renal haemangiopericytoma: the characteristics of a rare tumour. BJU Int. 2005; 95(7): 943-7.

11.Dragoumis D, Desiris K, Kyropoulou A, Malandri M, Assimaki A, Tsiftsoglou A. Hemangiopericytoma/solitary fibrous tumor of pectoralis major muscle mimicking a breast mass. Int J Surg Case Rep. 2013; 4(3): 338-41. doi: 10.1016/j.ijscr.2013.01.013.

12. Proietti A, Sartori C, Torregrossa L, et al. A case of metastatic haemangiopericytoma to the thyroid gland: Case report and literature review. Oncol Lett. 2012; 3(6): 1255-1258.

13. Krishnan M, Kumar KS, Sowmiya T. Hemangiopericytoma - the need for a protocol-based treatment plan. Indian J Dent Res. 2011; 22(3): 497. doi: 10.4103/0970-9290.87086.

14. Ramakrishna R, Rostomily R, Sekhar L, Rockhill J, and Ferreira M. Hemangiopericytoma: Radical resection remains the cornerstone of therapy. J Clin Neurosci. 2014; 21(4): 612-5. doi: 10.1016/j. jocn.2013.08.006.

15. Xu L, Ding G. Recurrent primary haemangiopericytoma of the bladder: A case report and literature review. Oncol Lett. 2014; 7(4): 11441146.

16. Sutton R, Hopper IP, Munson KW. Haemangiopericytoma of the bladder. Br J Urol. 1989; 63(5): 548-9.

17. Bagchi AG, Dasgupta A, Chaudhury PR. Haemangiopericytoma of urinary bladder. J Indian Med Assoc. 1993; 91(8): 211-2. 
18. Kibar Y, Uzar AI, Erdemir F, Ozcan A, Coban H, Seckin B. Hemangiopericytoma arising from the wall of the urinary bladder. Int Urol Nephrol. 2006; 38(2): 243-5.

19. Chaudhary A, Seenu V, Sedain G, et al. Hemangiopericytoma of renal pelvis--an unusual tumor in an adolescent. Urology. 2007; 70(4): 811. e13-4.

20. Brescia A, Pinto F, Gardi M, Maria vecchio F, Bassi PF. Renal hemangiopericytoma: case report and review of the literature.
Urology. 2008; 71(4): 755.e9-12. doi: 10.1016/j.urology.2007.10.066.

21. Mozafarpour S, Khorramirouz R, Tajali A, Salavati A, Kajbafzadeh AM. Surgically treated bladder hemangiopericytoma/solitary fibrous tumor: report of a 12-year asymptomatic follow-up. Int Urol Nephrol. 2014; 46(3): 483-6.

22. Prout MN, Davis HL. Hemangiopericytoma of the bladder after polyvinyl alcohol exposure. Cancer. 1977; 39(3): 1328-30. 